\title{
Vacuum stress around a topological defect
}

\author{
V. A. De Lorencia* and E. S. Moreira, Jr. ${ }^{a \dagger}$ \\ anstituto de Ciências, Universidade Federal de Itajubá, \\ Av. BPS 1303 Pinheirinho, 37500-903 Itajubá, MG, Brazil
}

\begin{abstract}
We show that a dispiration (a disclination plus a screw dislocation) polarizes the vacuum of a scalar field giving rise to an energy momentum tensor which, as seen from a local inertial frame, presents non vanishing off-diagonal components. The results may have applications in cosmology (chiral cosmic strings) and condensed matter physics (materials with linear defects).
\end{abstract}

It is fairly well known that a needle solenoid carrying a magnetic flux makes virtual charged particles to run around the solenoid inducing a non vanishing current density (see e.g. Ref [1]). We wish to consider what seems to be a gravitational (geometric) analogue of this AharonovBohm effect, by computing the vacuum expectation value of the energy momentum tensor of a massless and neutral scalar field far away from a dispiration.

Let us begin by presenting the geometry of the background (units are such that $c=\hbar=1$ ),

$d s^{2}=d t^{2}-d r^{2}-\alpha^{2} r^{2} d \theta^{2}-(d z+\kappa d \theta)^{2}$,

where the points labeled by $(t, r, \theta, z)$ and $(t, r, \theta+$ $2 \pi, z)$ are identified [23]. When $\alpha=1$ and $\kappa=0$ Eq (11) becomes the line element of the flat spacetime written in cylindrical coordinates. Borrowing terminologies in condensed matter physics, the parameters $\alpha$ and $\kappa$ correspond to a disclination and a screw dislocation, respectively. We should remark that Eq (1) may be associated with the gravitational background of certain chiral cosmic strings [4] (as has been suggested in Ref. [2]), as well as can describe (in the continuum limit) the effective geometry around a dispiration in an elastic solid (see Ref. [5] and references therein).

The definitions $\varphi:=\alpha \theta$ and $Z:=z+\kappa \theta$ lead to

$d s^{2}=d t^{2}-d r^{2}-r^{2} d \varphi^{2}-d Z^{2}$,

*delorenci@unifei.edu.br

${ }^{\dagger}$ moreira@unifei.edu.br which should be considered together with the peculiar identification

$(t, r, \varphi, Z) \sim(t, r, \varphi+2 \pi \alpha, Z+2 \pi \kappa)$.

Although Eq. (2) expresses the fact that the background is locally flat, due to Eq. (3) we cannot use Eq. (2) (which is a local statement) to infer that the global symmetries of the background are the same as those of the Minkowski spacetime (in this sense Eq. (2) is singular). In fact, Eq. (2) disguises a curvature singularity on the symmetry axis 2 (when $\kappa \neq 0$, in the context of the Einstein-Cartan theory, there is also a torsion singularity at $r=0$ [36]).

The vacuum expectation value of the energy momentum tensor is obtained by applying a differential operator to the renormalized scalar propagator around a dispiration (see e.g. Ref. [7]),

$$
\left\langle T^{\mu}{ }_{\nu}\right\rangle=i \lim _{x^{\prime} \rightarrow x} \mathcal{D}^{\mu}{ }_{\nu}\left(x, x^{\prime}\right) D^{(\alpha, \kappa)}\left(x, x^{\prime}\right) .
$$

We have recently obtained $D^{(\alpha, \kappa)}\left(x, x^{\prime}\right)$ (classical propagators have been considered in Ref [8) by using the Schwinger proper time prescription combined with the completeness relation of the eigenfunctions of the d'Alembertian operator 9]. Such eigenfunctions have the form $R(r) \chi(\varphi) \exp \{i(\nu Z-\omega t)\}$ which, by observing Eq. (3), leads to

$\chi(\varphi+2 \pi \alpha)=e^{-i 2 \pi \nu \kappa} \chi(\varphi)$.

This boundary condition is typical of the Aharonov-Bohm set up where $\nu \kappa$ is identified 
with the flux parameter $e \Phi / 2 \pi$. If we carry over to the four-dimensional context lessons from gravity in three dimensions 1011, it follows that the charge $e$ and the magnetic flux $\Phi$ should be identified with the longitudinal linear momentum $\nu$ and $2 \pi \kappa$, respectively [2].

When $\kappa / r \rightarrow 0$, Eq. (4) yields for the diagonal components the expressions of the vacuum fluctuations around an ordinary cosmic string $(\kappa=0)$ 12. Regarding the other components, the prescription in Eq. (4) kills off the dominant contribution in the renormalized propagator 9 , resulting that the subleading contribution yields two non vanishing off-diagonal components,

$$
\left\langle T^{\varphi} Z\right\rangle=\frac{i}{r^{2}} \lim _{x^{\prime} \rightarrow x} \partial_{\varphi} \partial_{Z} D^{(\alpha, \kappa)}\left(x, x^{\prime}\right)=\frac{\kappa}{r^{6}} B(\alpha),(6)
$$

and

$$
\left\langle T^{Z}{ }_{\varphi}\right\rangle=\frac{\kappa}{r^{4}} B(\alpha),
$$

where $B(\alpha)$ depends on the disclination parameter only [9. Unlike the diagonal components, $\left\langle T^{\varphi}{ }_{Z}\right\rangle$ and $\left\langle T^{Z}{ }_{\varphi}\right\rangle$ do not depend on the coupling parameter $\xi$.

When $\alpha=1, B=1 / 60 \pi^{2}$ which corresponds approximately to the value of $\alpha$ in the physics of formation of ordinary cosmic strings 13 .

It is instructive to display both disclination and screw dislocation effects in a same array. When $\xi=1 / 6$ (conformal coupling), for example, $\left\langle T^{\mu}{ }_{\nu}\right\rangle$ with respect to the local inertial frame [cf. Eq. (22)] can be cast into the form

$$
\left\langle T^{\mu}{ }_{\nu}\right\rangle=\frac{1}{r^{4}}\left(\begin{array}{cccc}
-A & 0 & 0 & 0 \\
0 & -A & 0 & 0 \\
0 & 0 & 3 A & \kappa B / r^{2} \\
0 & 0 & \kappa B & -A
\end{array}\right),
$$

where $A(\alpha):=\left(\alpha^{-4}-1\right) / 1440 \pi^{2}$, and which holds far away from the defect (and for $\alpha \neq 1$, when $\kappa \neq$ 0 ). [When $\kappa \neq 0$, by setting $\alpha=1$ in Eq. (8), $A$ vanishes and subleading contributions depending on $\kappa$ take over.]

Before closing we should report a discrepancy in the literature. Recently, works [1415] have appeared stating that, for $\alpha=1$, the subleading contribution in $\left\langle T^{0}{ }_{0}\right\rangle$ [cf. Eq. [8] is $\lambda \kappa^{2} / r^{6}$, where $\lambda$ is a certain constant. We have performed a rather detailed study showing that such a subleading contribution is $f(\kappa / r) \kappa^{2} / r^{6}$ instead, where $f(\kappa / r)$ diverges as $\kappa \rightarrow 0$ [although $\left.\lim _{\kappa \rightarrow 0} \kappa^{2} f(\kappa / r)=0\right]$.

\section{acknowledgments}

This work was partially supported by the Brazilian research agencies $\mathrm{CNPq}$ and FAPEMIG.

\section{REFERENCES}

1. E.M. Serebryanyi, Theor. Math. Phys. 64 (1985) 846.

2. D.V. Gal'tsov and P.S. Letelier, Phys. Rev. D 47 (1993) 4273.

3. K.P. Tod, Class. Quant. Grav. 11 (1994) 1331.

4. J.D. Bekenstein, Phys. Rev. D 45 (1992) 2794.

5. R.A. Puntigam and H.H. Soleng, Class. Quant. Grav. 14 (1997) 1129.

6. P.S. Letelier, Class. Quant. Grav. 12 (1995) 471.

7. N.D. Birrel and P.C.W. Davies, Quantum Fields in Curved Space, Cambridge University Press, Cambridge, England, 1982.

8. V.A. De Lorenci and E.S. Moreira, Jr., Phys. Rev. D 65 (2002) 107503.

9. V.A. De Lorenci and E.S. Moreira Jr., hep-th/0301194

10. P.S. Gerbert and R. Jackiw, Commun. Math. Phys. 124 (1989) 229.

11. P.S. Gerbert, Nucl. Phys. B 346 (1990) 440.

12. M.E.X. Guimarães and B. Linet, Commun. Math. Phys. 165 (1994) 297.

13. A. Vilenkin and E.P.S. Shellard, Cosmic Strings and Other Topological Defects, Cambridge University Press, Cambridge, England, 1994.

14. I. Pontual and F. Moraes, Philos. Mag. A 78 (1998) 1073.

15. T.M. Helliwell, D.A. Konkowski, and V. Arndt, Gen. Rel. Grav. 35 (2003) 79. 